\title{
HMGB1 translocation is involved in the transformation of autophagy complexes and promotes chemoresistance in leukaemia
}

\author{
QIAN KONG ${ }^{1,2}$, LU-HONG XU ${ }^{1,2}$, WEI XU ${ }^{2}$, JIAN-PEI FANG ${ }^{1,2}$ and HONG-GUI XU ${ }^{1,2}$ \\ ${ }^{1}$ Guangdong Provincial Key Laboratory of Malignant Tumour Epigenetics and Gene Regulation, Sun Yat-Sen \\ Memorial Hospital, Sun Yat-Sen University, Guangzhou, Guangdong; ${ }^{2}$ Department of Pediatrics, \\ Sun Yat-Sen Memorial Hospital, Sun Yat-Sen University, Guangzhou, Guangdong, P.R. China
}

Received March 6, 2015; Accepted April 17, 2015

DOI: 10.3892/ijo.2015.2985

\begin{abstract}
Acute lymphoblastic leukaemia (ALL) is a common paediatric cancer and is among the most curable cancers. However, the acquisition of drug resistance is a significant obstacle to the achievement of favourable outcomes, and autophagy is regarded as a mechanism that underlies chemoresistance. In this study, RT-qPCR was used to measure the expression of HMGB1 and Beclin1 in bone marrow mononuclear cells. A CCK-8 test was conducted to assess cell viability. Western blot, immunofluorescence and transmission electron microscopic analyses were performed to evaluate the autophagy levels. Immunoprecipitation analysis was performed to detect protein-protein interactions in the autophagy complexes. We found that HMGB1 expression correlated with the clinical status of ALL. In vitro, anticancer agent-induced cytotoxic effects were associated with autophagy-related drug resistance, and these effects were ameliorated by FIP200 depletion or the application of autophagy inhibitors. Moreover, the Ulk1-Atg13-FIP200 complex, which promotes HMGB1 trafficking, acted upstream of the HMGB1-Beclin1 and PI3KC3-Beclin1 complexes and played a critical role in autophagy. Targeting the transformation of autophagic complexes or HMGB1 translocation may suppress autophagy and consequently overcome chemoresistance in leukaemia.
\end{abstract}

\section{Introduction}

Leukaemia is the most common type of cancer in children. Chemoresistance represents a great obstacle to leukaemia therapy that might be responsible for refractory and relapsing cases. Various mechanisms underlying drug resistance have been recognised, such as those involving drug exporters, altered drug target sites, enhanced effectiveness of DNA repair mechanisms, altered pharmacokinetics, the downregulation of

Correspondence to: Professor Jian-Pei Fang, Department of Pediatrics, Sun Yat-Sen Memorial Hospital of Sun Yat-Sen University, 107 Yanjiang West Road, Guangzhou 510120, Guangdong, P.R. China E-mail: jpfang2005@163.com

Key words: acute lymphoblastic leukaemia, childhood leukaemia, autophagy, chemoresistance, HMGB1 apoptosis and resistant tumour stem cells (1). In recent years, autophagy, which plays an intricate role in cell death and survival, has also been considered as a potential mechanism underlying resistance to chemotherapy, radiation therapy and immunotherapy in cancer cells (2-5).

During the autophagic process, energy production is maintained via the degradation of cellular components and the recycling of organelles. The mammalian autophagic process begins with autophagosome formation, which can be separated into three steps: initiation and nucleation, elongation, maturation. During the initiation stage, the Ulk1Atg13-FIP200 complex is activated by an autophagic signal and participates in autophagosome formation $(6,7)$. Then, the class III PI3K (PI3KC3) complex, which is composed of PI3KC3, Beclin1, p150 and ATG14, plays an essential role in isolation membrane nucleation (8). Subsequently, the membrane is elongated by a series of autophagy-related proteins (ATG), with the transition from LC3-I to LC3-II. At last, the autophagosome is maturated with the action of LC3-II and Beclin1 (9,11).

In the absence of autophagy stimulation, Beclin1 binds to Bcl-2 and remains stable. Upon sensing cell stress, high mobility group box 1 (HMGB1), which is primarily localised to the nucleus, translocates to the cytoplasm and promotes the phosphorylation of $\mathrm{Bcl}-2$, thereby promoting the escape of Beclin1 from Bcl-2 and subsequently facilitating PI3KC3Beclin1 complex assembly $(12,13)$.

As critical effector of autophagosome formation, the Ulk13-FIP200 complex, the PI3KC3 complex and the HMGB1 complex are not independent modules. In addition, the translocation of HMGB1 is a crucial molecular event during autophagy. However, the association between the activities of these complexes and the function of HMGB1 translocation, specifically in leukaemia, have yet to be clearly elucidated.

In the present study, we demonstrated that both the Ulk1Atg13-FIP200 complex and the HMGB1-Beclin1 complex acted as critical regulators of autophagy. Their core components, FIP200 and HMGB1, were essential for autophagy-related drug resistance in leukaemia. The Ulk1-Atg13-FIP200 complex acted upstream of the HMGB1-Beclin1 and PI3KC3-Beclin1 complexes. Additionally, we first discovered that the translocation of HMGB1 represented a decisive step in the autophagic process and was involved in a regulatory relationship between 
Ulk1-Atg13-FIP200 and HMGB1-Beclin1 complex. These findings revealed that the inhibition of autophagy may augment chemotherapy and may help to overcome drug resistance.

\section{Materials and methods}

Subjects and cell isolation. This study was approved by the Ethics Committee of the Sun Yat-Sen Memorial Hospital of Sun Yat-Sen University. Written informed consent was obtained from all the participants or their parents.

Bone marrow samples were collected from patients aged from 0 to 14 years who suffered from childhood acute lymphoblastic leukaemia (ALL) or a non-malignant blood disease. In total, 30 subjects with B-cell ALL (12 patients in the primary phase, 12 in the complete remission phase who were still undergoing treatment, 3 patients in the complete remission phase who had completed their treatment course and 3 in the relapse or non-remission phase) and 8 subjects with non-malignant blood disease were enrolled. The diagnoses of ALL were based on morphologic and flow cytometric analyses of the immunophenotype, and the stratification of ALL was based on the IC-BFM 2002 criteria for ALL. Bone marrow mononuclear cells (BMMCs) were isolated via Ficoll (MP Biomedicals, Santa Ana, CA, USA) density gradient centrifugation (14).

Quantitative real-time PCR (RT-qPCR). Total RNA was isolated from BMMCs using TRIzol reagent (Takara Bio Inc., Otsu, Shiga, Japan) and then reverse-transcribed into cDNA. The sequences of primers used were as follows: GAPDH: forward, 5'-GGTCGGAGTCAACGGATTTGGTCG-3' and reverse, 5'-CCTCCGACGCCTGCTTCACCAC-3'; for HMGB1: forward, 5'-TTTCAAACAAAGATGCCACA-3' and reverse, 5'-GTTCCCTAAACTCCTAAGCAGATA-3'; for Beclin1: forward, 5'-ATCCTGGACCGTGTCACCATCCAGG-3' and reverse, 5'-GTTGAGCTGAGTGTCCAGCTGG-3'; for FIP200: forward, 5'-TCAGTAAGTTGCGCAGTAGCA-3' and reverse, 5'-TTCAGGTGCACAAGCTCCAT-3'. Reactions were carried out in a Roche Light Cycler 480 system (Roche Diagnostics GmbH, Mannheim, Germany) with a SYBR Premix ExTaq kit (Takara Bio Inc., Otsu, Shiga, Japan). With the $2^{-\Delta \Delta C t}$ method, Data were normalized to GAPDH expression. The control group was set as 1 .

Cell culture, antibodies and reagents. The human leukaemia cell line, Jurkat and Reh, were purchased from the Type Culture Collection of the Chinese Academy of Sciences, Shanghai, China and cultured in RPMI-1640 medium (Gibco, Grand Island, NY, USA) with 10\% FBS (Hyclone, Logan, UT, USA) in a humidified incubator with $5 \% \mathrm{CO}_{2}$ and $95 \%$ air (15).

The antibodies to FIP200, ULK1, mAtg13, PI3KC3, Phospho-ULK1 (Ser555) and Rabbit (DA1E) mAb IgG XPTM Isotype Control were obtained from Cell Signaling Technology (Danvers, MA, USA). The antibodies to LC3 were obtained from Sigma-Aldrich (St. Louis, MO, USA). The antibodies to HMGB1, Beclin1, Bcl-2 and P62 were obtained from Abcam (Cambridge, UK). The antibodies to actin, GAPDH and tubulin were obtained from EarthOx Life Science (Millbrae, CA, USA). All secondary antibodies were purchased from Bioss (Beijing Biosynthesis Biotechnology
Co., Ltd.). Vincristine (VCR), daunorubicin (DNR), 3-methyladenine (3-MA), chloroquine (CQ) and ethyl pyruvate (EP) were purchased from Sigma-Aldrich.

Lentivirus infection and cell sorting. Lentivirus with shRNA was purchased from GenePharma (Shanghai, China). Transfection was conducted with lentivirus using $5 \mu \mathrm{g} / \mathrm{ml}$ polybrene and selected with $1 \mu \mathrm{g} / \mathrm{ml} 1$ puromycin under the instruction of GenePharma Recombinant Lentivirus Operation Manual (16). The interference efficiency was evaluated by RT-qPCR and western blotting. We selected the best sequence which processed the highest interference efficiency out from 3 different sequences. The optimal target sequence for FIP200 was 5'-GCCGTCAAACTATTGCCAAAC-3', for HMGB1 was 5'-CCCGTTATGAAAGAGAAATGA-3' and for control was 5'-TTCTCCGAACGTGTCACGTTTC-3'.

Cell viability analysis. Cells were plated in 96-well plate at a density of $\sim 6 \times 10^{4}$ cells/well. Cell viability was measured after medication treatments by CCK-8 test (Dojindo Molecular Technologies Inc., Kumamoto, Kyushu, Japan) according to the manufacturer's instructions.

Western blot analysis. Cells with different treatments were collected and lysed. The cell lysate was separated by $8 \%$ $(10,12$ and $15 \%)$ sodium dodecyl sulfate-polyacrylamide gel electrophoresis (SDS-PAGE) and electrophoretically transferred onto polyvinylidene fluoride membrane (Millpore, Billerica, MA, USA). The membrane was blocked with 5\% non-fat milk, and then incubated with the primary antibodies at a dilution of 1:1,000 overnight at $4^{\circ} \mathrm{C}$. After rinsing, the membranes were incubated for $1 \mathrm{~h}$ at room temperature with secondary antibodies at a dilution of 1:1,000 and then washed with TBST $(15,17,18)$. The membranes were detected with enhanced chemiluminescence reagent (Millpore) by G: BOX XT4 (Syngene, Cambridge, UK). The expressions of the target proteins were quantified by Quantity One version 4.6.2.

Immunoprecipitation analysis. Immunoprecipitation (IP) analysis was performed by Pierce Classic IP kit (Pierce Biotechnology, Rockford, IL, USA) according to the manufacturer's protocol. In brief, cells were collected and lysed by ice cold IP lysis buffer. Then the protein was pre-cleared with the control agarose resin at $4^{\circ} \mathrm{C}$ for $1 \mathrm{~h}$. The pre-cleared cell lysate was incubated with the Rabbit (DA1E) $\mathrm{mAb} \operatorname{IgG} \mathrm{XP}^{\mathrm{TM}}$ Isotype Control or specific antibodies overnight at $4^{\circ} \mathrm{C}$ to form the immune complex and then with protein $\mathrm{A} / \mathrm{G}$ plus agarose for $1 \mathrm{~h}$ at $4^{\circ} \mathrm{C}$ to capture the immune complex. After washed the resin with wash buffer, the immune complex was eluted at $100^{\circ} \mathrm{C}$ for $10 \mathrm{~min}$. The eluate was collected and subjected to SDS-PAGE for immunoblot analysis $(15,18)$. The CleanBlot IP Detection reagent (Pierce Biotechnology) was used as secondary antibody at a dilution of 1:1,000 for detecting the target proteins without interference from denatured $\operatorname{IgG}$ in western blot analysis.

Immunofluorescence analysis. Cells were collected and fixed in $4 \%$ formaldehyde for $20 \mathrm{~min}$, permeabilized in $0.3 \%$ Triton X-100 for $5 \mathrm{~min}$ and incubated in blocking buffer for 
A

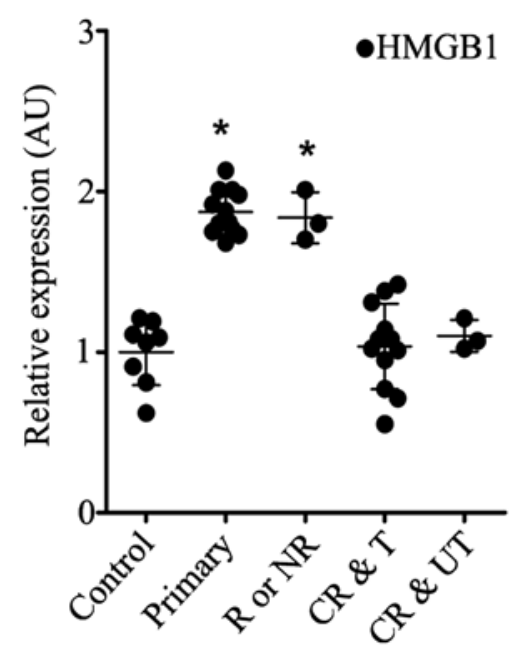

B

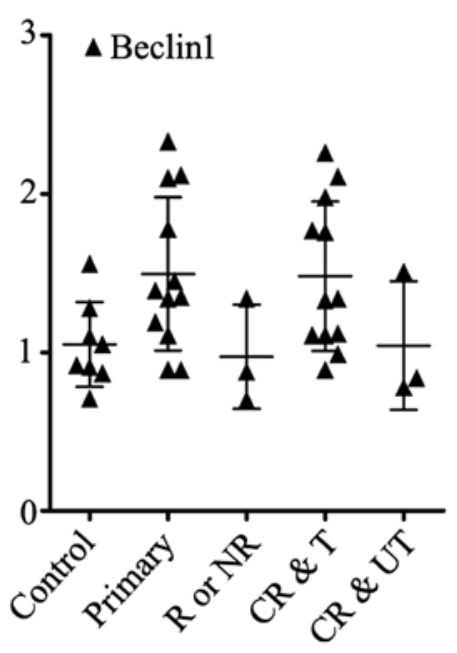

Figure 1. HMGB1 expression correlates with the stage of childhood acute lymphoblastic leukaemia. Total mRNA was extracted from the patient BMMCs, and the expression levels were determined via RT-qPCR. The average expression level in the control subjects was normalised to 1 . Each symbol represents the relative expression level in each sample. (A) The relative expression levels of HMGB1 ("p<0.005). (B) The relative expression levels of Beclin1. Control, control subjects with a non-malignant blood disease $(n=8)$. Primary, subjects with primary leukaemia $(n=12)$. $R$ or NR, subjects with relapsed or non-remitted leukaemia $(\mathrm{n}=3)$. $\mathrm{CR}$ and $\mathrm{T}$, subjects with complete remission of leukaemia or those who were under treatment $(\mathrm{n}=12)$. CR and UT, subjects exhibiting complete remission leukaemia or who had completed their treatment course $(n=3)$. AU, arbitrary unit.

$1 \mathrm{~h}$. Then cells were incubated overnight at $4^{\circ} \mathrm{C}$ with primary antibodies. After rinsing, cells were incubated with FITC- or Cy3-conjugated secondary antibodies for $1 \mathrm{~h}$ at room temperature in the dark and then with DAPI for $3 \mathrm{~min}$. After rinsing, cells were resuspended. Several drops of cell suspension were added in a laser confocal Petri dish $(12,19)$. The images were captured by a Carl Zeiss LSM 710 (Carl Zeiss Inc., Oberkochen, Germany).

Transmission electron microscopic analysis. Cells were fixed with $2 \%$ paraformaldehyde and $2.5 \%$ glutaraldehyde in $0.1 \mathrm{~mol} / \mathrm{l}$ phosphate buffer ( $\mathrm{pH} 7.4)$, followed by $1 \% \mathrm{OsO}_{4}$. After dehydration, thin sections were stained with uranyl acetate and lead citrate for observation by Tecnai G2 Spirit Twin electron microscope (FEI, Hillsboro, OR, USA). Transmission electron microscopic (TEM) assessment of autophagosome-like structures was carried out as previously described $(19,20)$.

Statistical analysis. Data are shown as mean \pm standard deviation (SD). The difference among groups was examined using the Student's t-test or ANOVA in measurement data and Fisher's exact probabilities in small size enumeration data. The Pearson product-moment correlation method was used to analyze the linear correlation between two groups. A value of $\mathrm{p}<0.05$ was regarded as statistically significant. All statistical analyses were conducted by SPSS version 18.0.

\section{Results}

HMGB1 expression correlates with the childhood acute lymphoblastic leukaemia stage. In total, 30 children with B-cell ALL and 8 control subjects with a non-malignant blood disease were included in the present study.

As shown in Fig. 1A, HMGB1 was upregulated in the BMMCs obtained from the patients with primary, relapsed or non-remission leukaemia, whereas its expression level in the BMMCs from the ALL patients who exhibited complete remission was as low as that from those with a non-malignant blood disease. The expression level of Beclin1 was nearly identical between the groups (Fig. 1B). The expression levels of HMGB1 and Beclin1 appeared to be irrelevant to risk stratification ( $\mathrm{p}>0.05$, data not shown).

The inhibition of autophagy and FIP200 depletion increases the sensitivity of leukaemia cells to chemotherapy. DNR and VCR are basic drugs used for leukaemia therapy. As shown in Fig. 2A, both drugs caused striking damage to Jurkat and Reh cells in a dose-dependent manner, and this trend was particularly apparent in the Reh cells. Interestingly, in the presence of 3-methyladenine (3-MA), an inhibitor of earlyphase autophagy, the cytotoxic effect was much stronger and cell viability was more sharply reduced (Fig. 2B). This result suggested that the inhibition of autophagy may augment the lethality of anticancer agents.

To explore the potential role of FIP200, a key protein involved in autophagy, a target-specific shRNA against FIP200 was transfected into Jurkat and Reh cells. This treatment resulted in a significant decrease in FIP200 at both the mRNA and protein levels (Fig. 2C). Cell viability analysis showed that the silencing of FIP200 mimicked the inhibitory effect of 3-MA and rendered the leukaemia cells more sensitive to DNR and VCR (Fig. 2A).

Silencing of FIP200 suppresses chemotherapy-induced autophagy in leukaemia cells. Autophagy is a highly dynamic, multi-step process. To assess the entire autophagic process, we measured autophagosomes containing LC3 puncta using immunofluorescence staining and analysed the LC3-II/I ratio via western blot analysis. Additionally, we observed ultrastructural changes via electron microscopy and assessed 

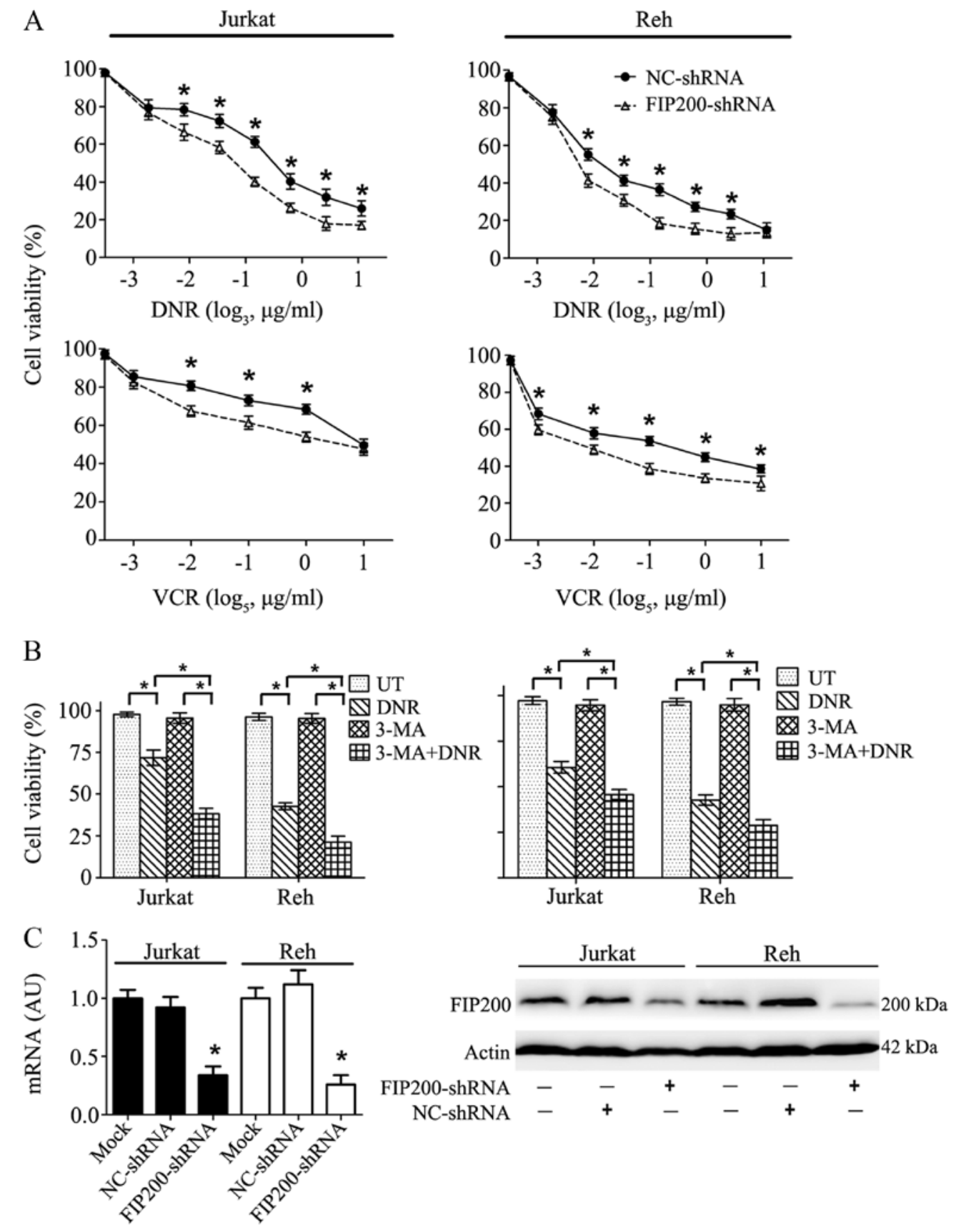

Figure 2. Autophagy inhibitor treatment and FIP200 depletion increased the sensitivity of leukaemia cells to chemotherapy. (A) Chemotherapeutic drugs exerted damaging effects on the leukaemia cells in a dose-dependent manner, and this effect was exacerbated by FIP200 depletion. Jurkat and Reh cells were treated with DNR $(0,0.05,0.1,0.2,0.4,0.8,1.6 \mu \mathrm{g} / \mathrm{ml}$, or $3.2 \mu \mathrm{g} / \mathrm{ml})$ or VCR $(0,0.008,0.04,0.2,1$, or $5 \mu \mathrm{g} / \mathrm{ml})$ for $24 \mathrm{~h}$. Cell viability was analysed using a CCK-8 kit. (B) The inhibition of autophagy ameliorated drug resistance. Jurkat and Reh cells were treated with DNR $(0.4 \mu \mathrm{M})$ or VCR $(1 \mu \mathrm{M})$ for $24 \mathrm{~h}$ with or without 3-MA (5 mM). Cell viability was analysed using a CCK-8 kit $\left(n=3,{ }^{*} \mathrm{p}<0.001\right)$. (C) Jurkat and Reh cells were transfected with control or FIP200 shRNA. Then, the mRNA and protein levels of FIP200 were assessed via RT-qPCR and western blot analysis, respectively ( $\mathrm{n}=3$, " $\mathrm{p}<0.05)$. UT, untreated group. The viability of the control cells was normalised to $100 \%$.

autophagic flux based on lysosomal turnover and the P62 level via western blot analysis $(19,21,22)$.

Based on western blot analysis, we found that silencing FIP200 suppressed the chemotherapy-induced high expression of LC3-II and the corresponding degradation of P62 (Fig. 3A and B). The accumulation of LC3-II in the mock-treated and control cells was enhanced in the presence of the lysosomal inhibitor chloroquine (CQ) in the presence or absence of stimuli, but this effect was not observed in the FIP200 shRNA-treated cells (Fig. 3A). These changes demonstrated that the accumulation of LC3-II was not due to its decreased degradation but rather increased autophagic flux. However, the changes of LC3-II and P62 were not observed in the presence of 3-MA (Fig. 3B). Based on immunofluorescence analysis, the change in endogenous LC3 puncta was consistent with the results of western blot analysis in both cell lines (Fig. 3C). Moreover, ultrastructural analysis revealed that the FIP200 shRNA-treated cells exhibited fewer autophagosomes during chemotherapy compared with the control shRNA-treated cells (Fig. 3D). 
A

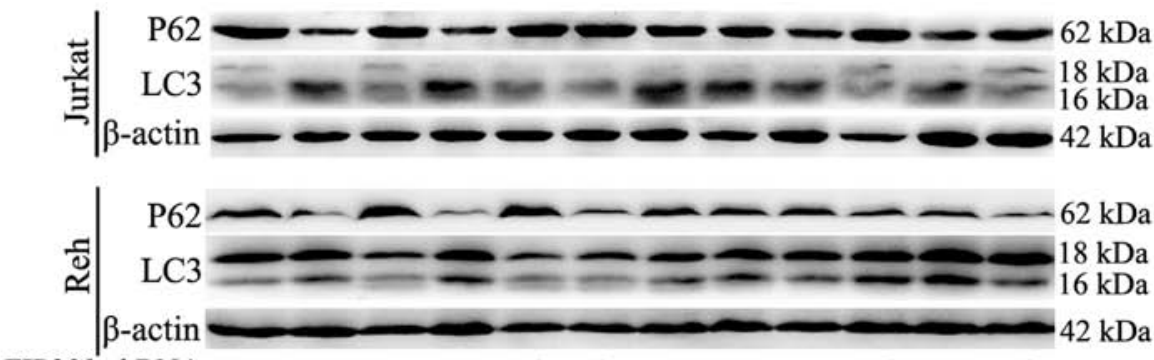

FIP200-shRNA - -+-+-+-+

NC-shRNA -+ \pm+-+-

$\mathrm{DNR}-+ \pm+\frac{+}{\mathrm{CQ}}- \pm+\frac{+}{+}+ \pm+$

B

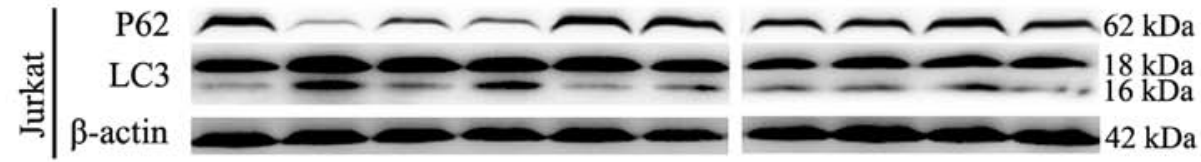

$\mathrm{C}$
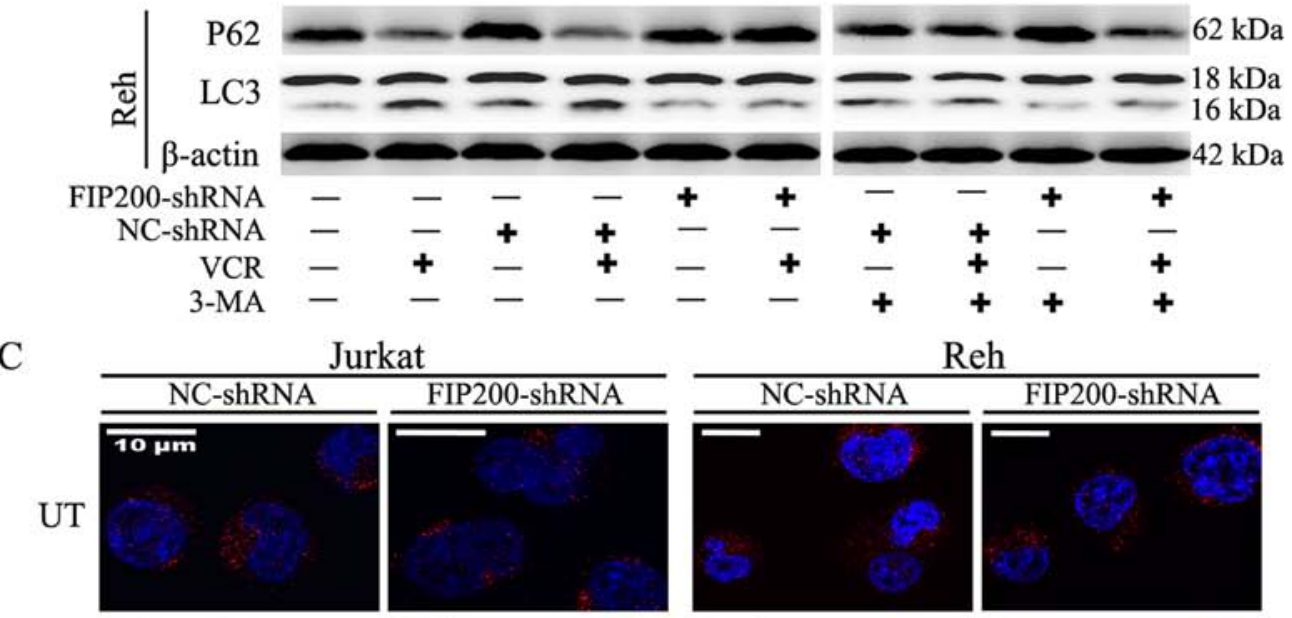

Jurkat
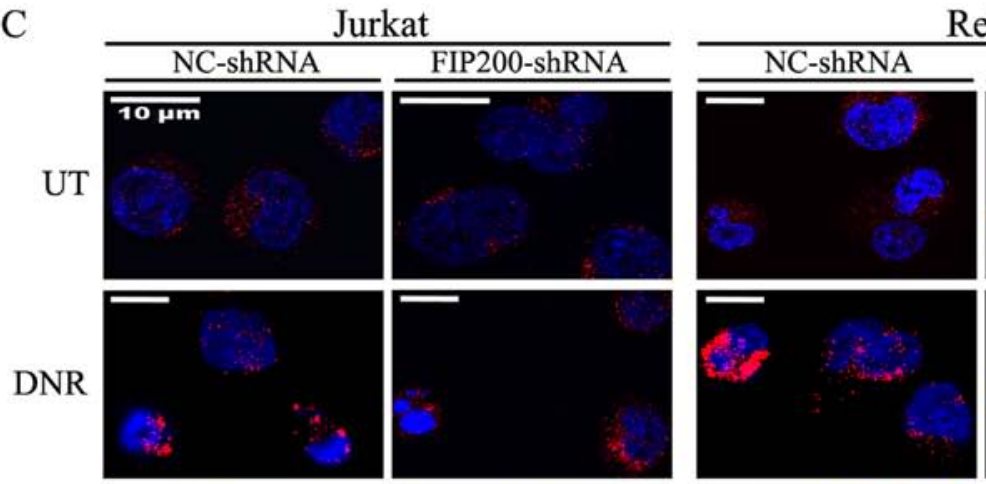

Reh
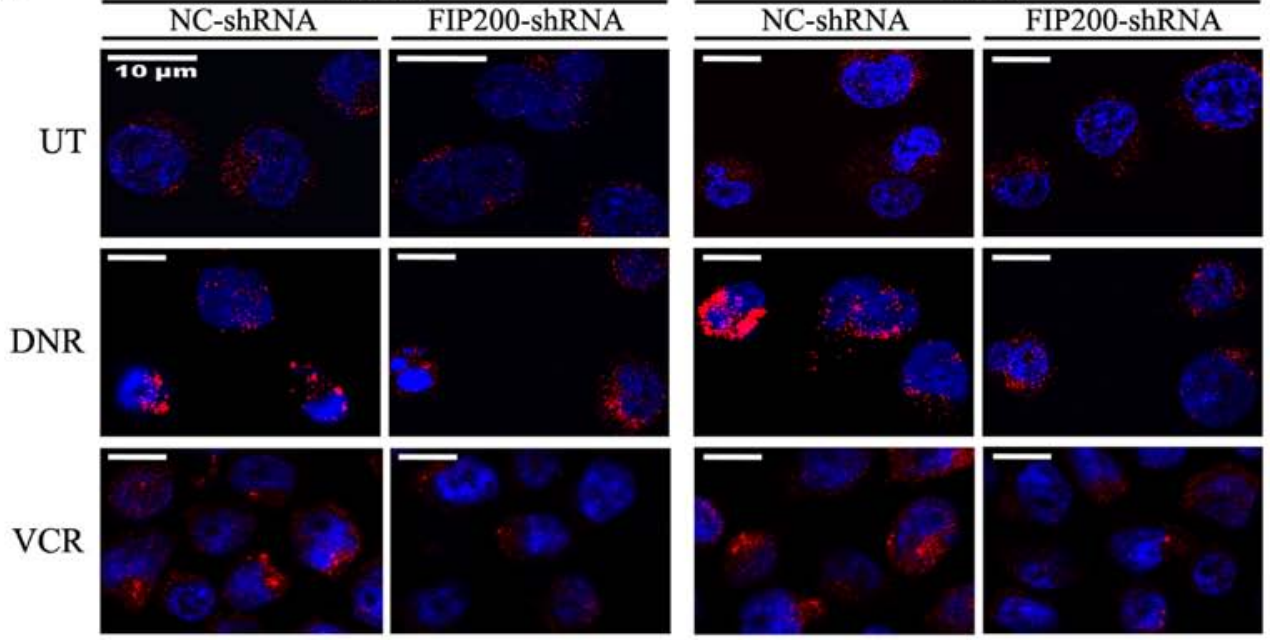

LC3/Nucleus

$\mathrm{D}$

Jurkat

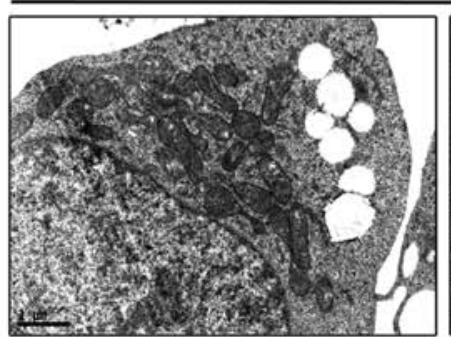

NC-shRNA

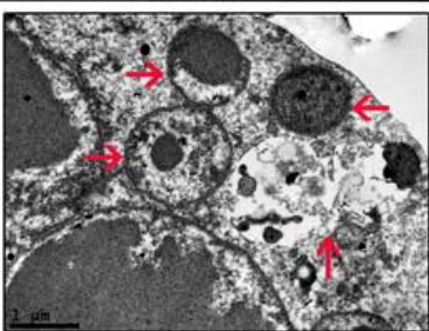

NC-shRNA+DNR

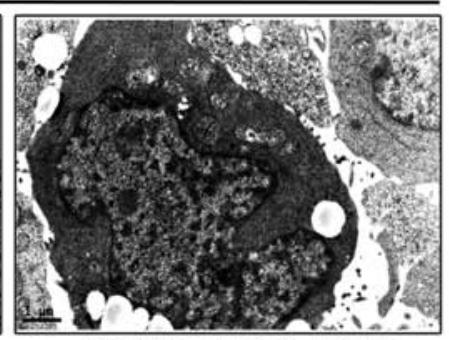

FIP200-shRNA+DNR

Figure 3. FIP200 depletion suppressed autophagy in leukaemia cells. (A) Jurkat and Reh cells were transfected with control or FIP200 shRNA followed by DNR $(0.4 \mu \mathrm{M})$ treatment in the presence or absence of CQ $(25 \mu \mathrm{M})$ for $24 \mathrm{~h}$. The cells were subjected to western blot analysis of LC3-II/I and p62 expression. Actin was used as a loading control. (B) Jurkat and Reh cells were transfected with control or FIP200 shRNA followed by VCR (1 $\mu$ M) treatment in the presence or absence of 3-MA $(5 \mathrm{mM})$ for $24 \mathrm{~h}$. The cells were subjected to western blot analysis of LC3-II/I and p62 expression. Actin was used as a loading control. (C) Jurkat and Reh cells were transfected with control or FIP200 shRNA followed by DNR $(0.4 \mu \mathrm{M})$ or VCR ( $1 \mu \mathrm{M})$ treatment for $24 \mathrm{~h}$. The cells were subjected to confocal microscopic analysis to measure the LC3 puncta using an anti-LC3 antibody (red, LC3; blue, nucleus). (D) Jurkat cells were transfected with control or FIP200 shRNA followed by treatment with or without DNR $(0.4 \mu \mathrm{M})$ for $24 \mathrm{~h}$. Then, the cells were subjected to transmission electron microscopy to observe autophagosome-like structures (indicated by the red arrows). UT, untreated group. 
A
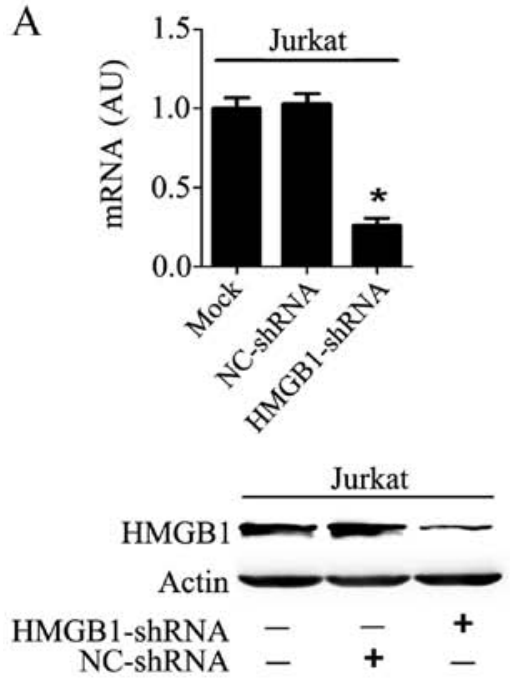

B
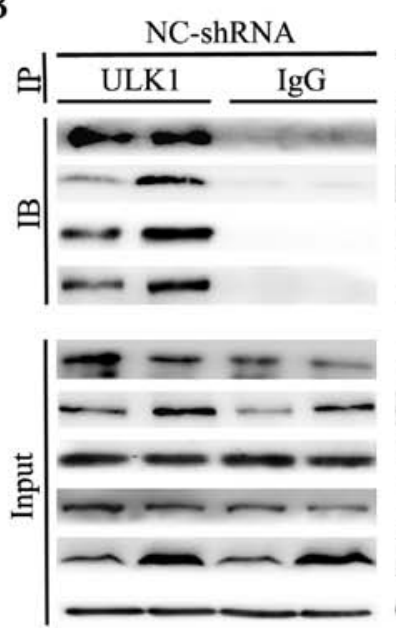

UT DNR UT DNR

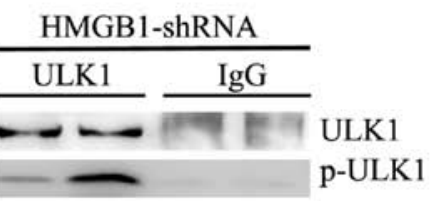

Atg-13
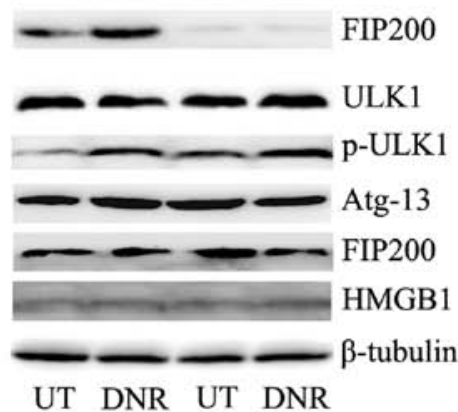

$\mathrm{C}$
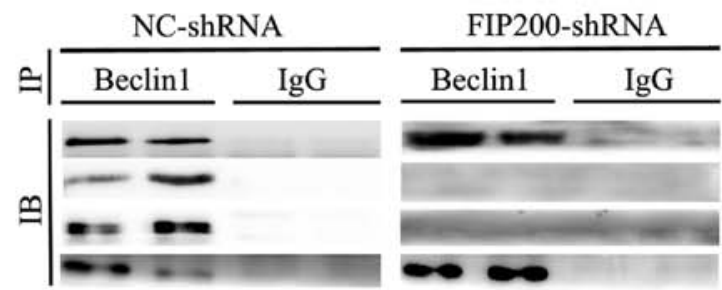

HMGB1-shRNA
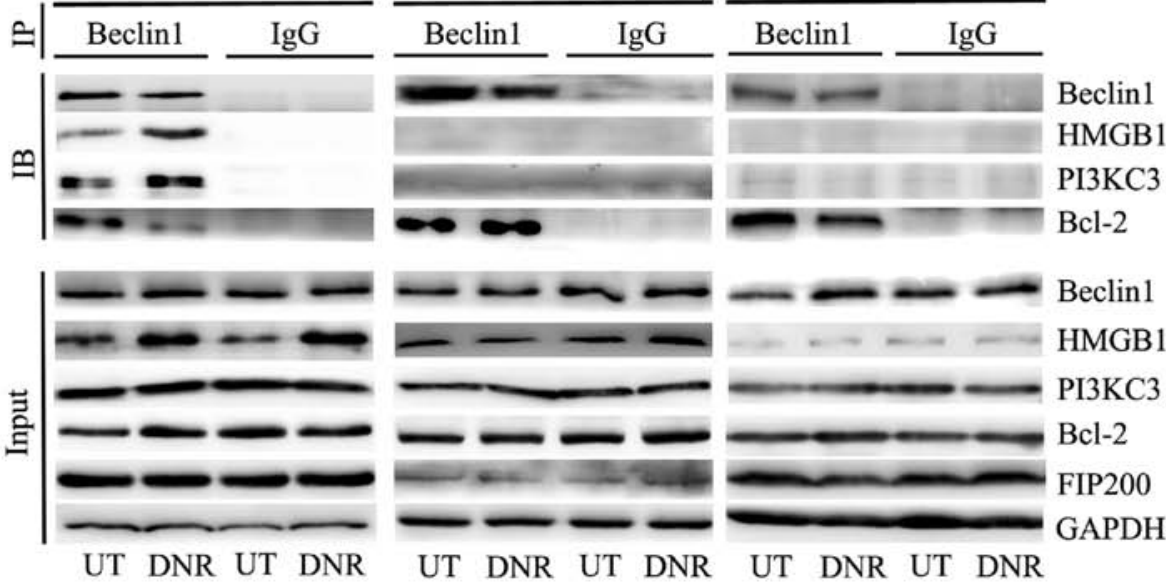

UT DNR UT DNR

UT DNR UT DNR

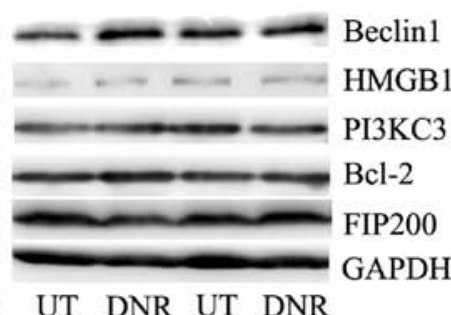

$\mathrm{D}$

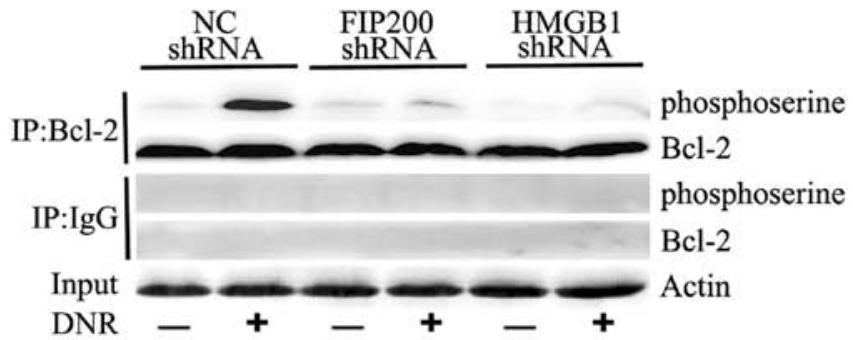

Figure 4. The ULK1-mAtg13-FIP200 complex functions upstream of the HMGB1-Beclin1 complex. (A) Jurkat cells were transfected with control or HMGB1 shRNA. The mRNA and protein levels of HMGB1 were assessed via RT-qPCR and western blot analysis, respectively $\left(\mathrm{n}=3\right.$, $\left.{ }^{*} \mathrm{p}<0.05\right)$. (B) Jurkat cells were transfected with control or HMGB1 shRNA and treated with or without DNR $(0.4 \mu \mathrm{M})$ for $24 \mathrm{~h}$. The resulting immunocomplexes were analysed via immunoprecipitation and western blot analysis using specific antibodies. Tubulin was used as a loading control. (C and D) Jurkat cells were transfected with control, FIP200 or HMGB1 shRNA and then treated with or without DNR $(0.4 \mu \mathrm{M})$ for $24 \mathrm{~h}$. The cell lysates were subjected to immunoprecipitation followed by western blot analysis. GAPDH and actin were used as loading controls.

The data shown in Figs. 2 and 3 demonstrate that in leukaemia, the anticancer agent-induced cytotoxic effects were due to the autophagy-related drug resistance, and these effects were ameliorated by the depletion of FIP200 or the application of an autophagy inhibitor.

The activation of the ULK1-mAtg13-FIP200 complex served as an upstream signal to HMGB1-Beclin1 formation during autophagy. We transfected Jurkat cells with HMGB1 shRNA, which resulted in a prominent decrease in the mRNA and protein expression of HMGB1 (Fig. 4A). As shown in Fig. 4B, the phosphorylation of ULK1 at Ser555, which plays a critical role in responding to autophagic stimuli, was significantly increased after anticancer agent treatment. Immunoprecipitation analysis indicated that silencing HMGB1 did not affect ULK1-mAtg13-FIP200 complex assembly or ULK1 phosphorylation following DNR treatment.

Conversely, the absence of FIP200 did inhibit the assembly of the HMGB1-Beclin1 complex or the subsequent formation of the PI3KC3-Beclin1 complex after chemotherapy stimulation (Fig. 4C). In the control shRNA-treated Jurkat cells, Beclin1 separated from Bcl-2 after DNR treatment and subse- 
A
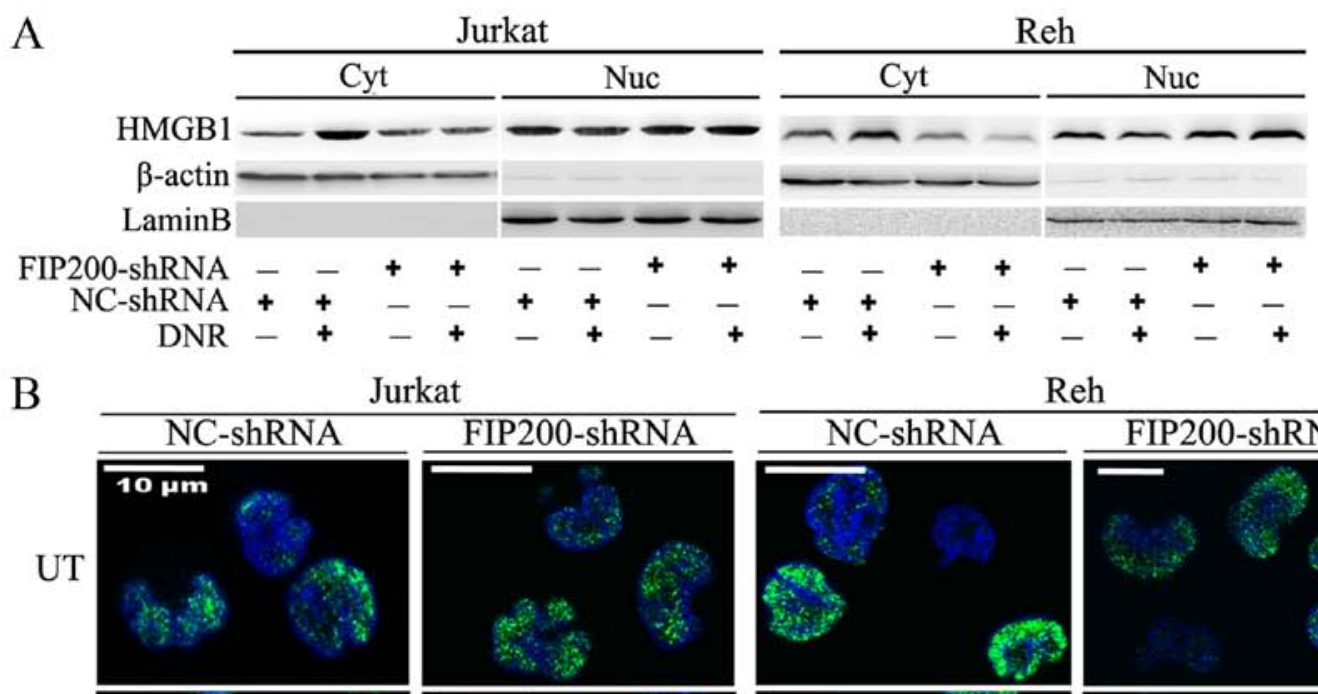

Jurkat
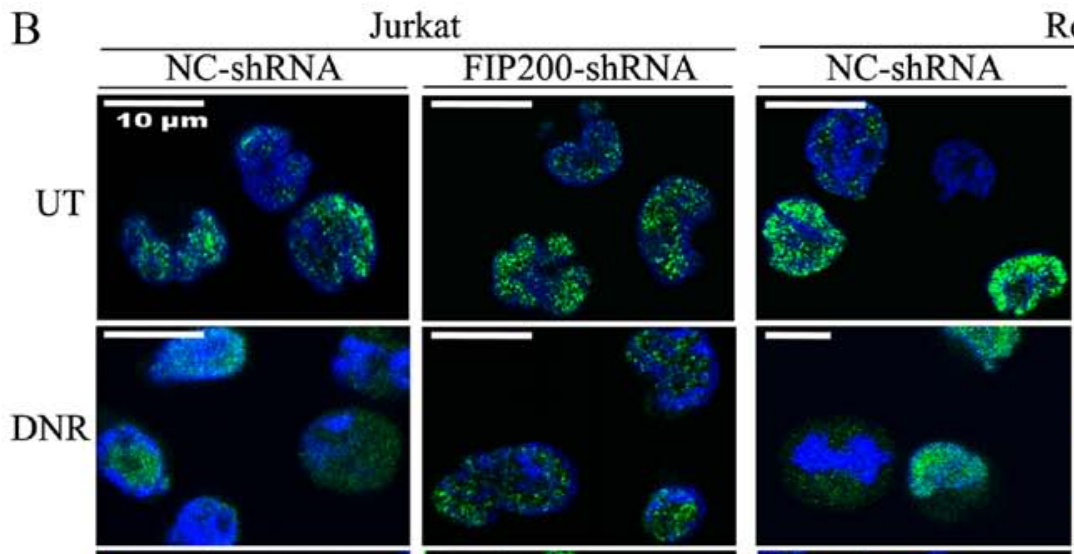

Reh
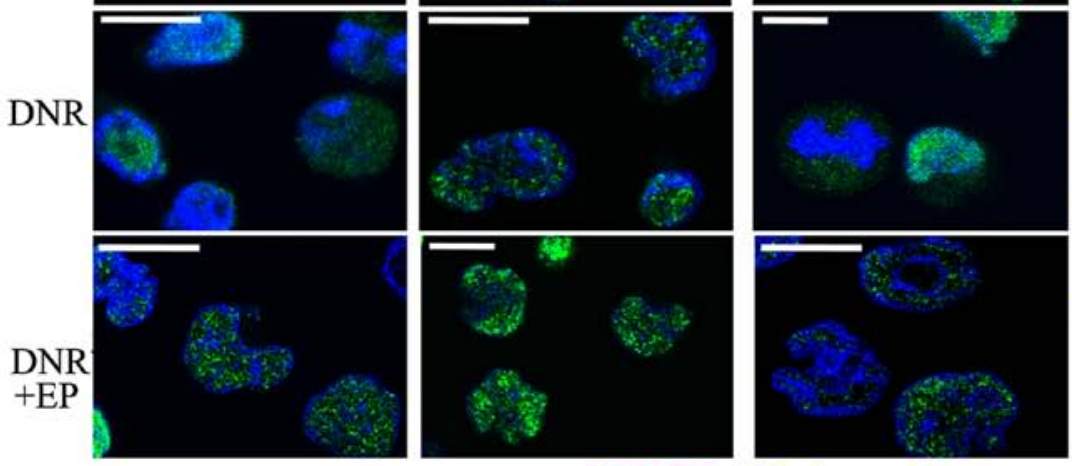

FIP200-shRNA
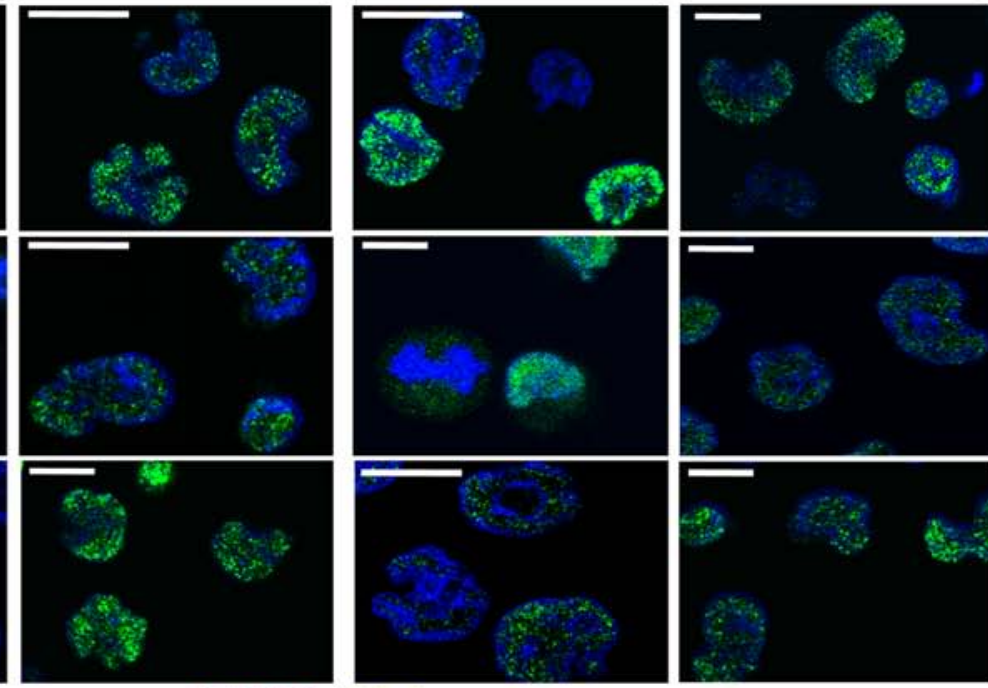

HMGB1 / Nucleus

$\mathrm{C}$

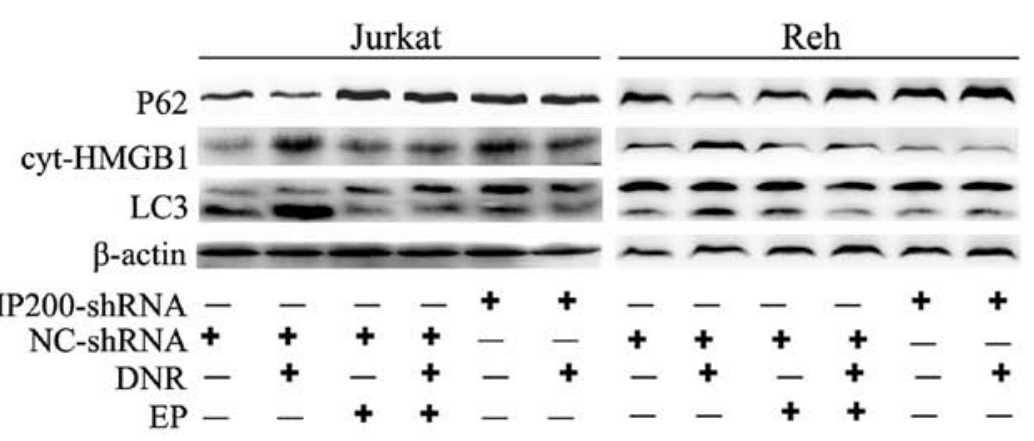

D

Jurkat

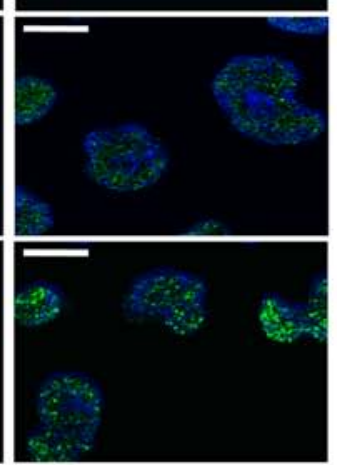

$\mathrm{E}$

$\square \mathrm{UT} \quad$ 目EP

国 DNR $\square \mathrm{DP}+\mathrm{DNR}$

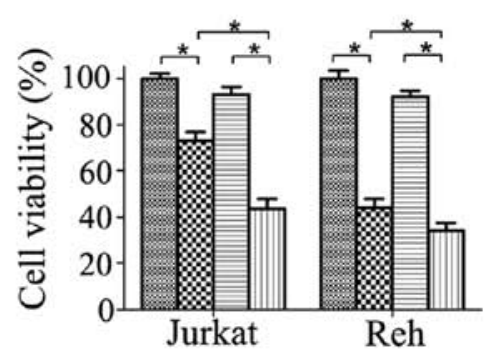

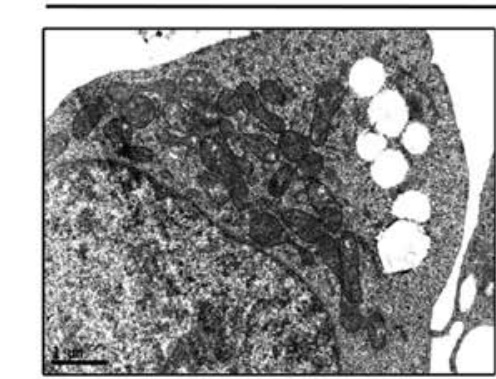

NC-shRNA

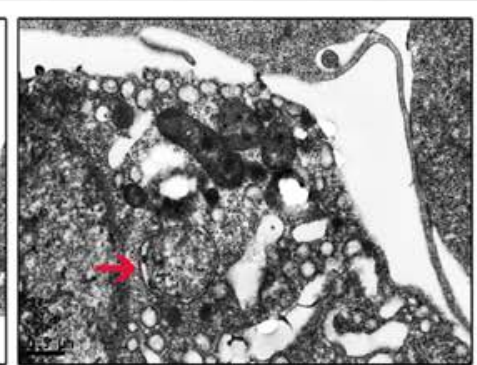

NC-shRNA +DNR +EP

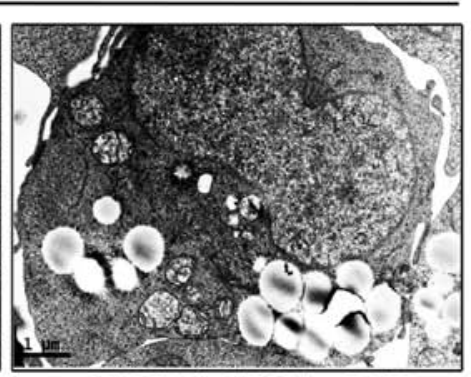

HMGB1-shRNA +DNR

Figure 5. The translocation of HMGB1 is essential for autophagy-mediated chemoresistance in leukaemia cells. (A) Jurkat and Reh cells were transfected with control or FIP200 shRNA and then treated with or without DNR $(0.4 \mu \mathrm{M})$ for $24 \mathrm{~h}$. The cell lysates were separated into the cytosolic and nuclear fractions, which were subjected to western blot analysis of HMGB1 expression. Actin and lamin-B were used as loading controls for cytosolic and nuclear proteins, respectively. (B) Jurkat and Reh cells were transfected with control or FIP200 shRNA followed by DNR $(0.4 \mu \mathrm{M})$ treatment for $24 \mathrm{~h}$. The cells were subjected to confocal microscopic analysis to detect the localisation of HMGB1 (green, HMGB1; blue, nucleus). (C) Jurkat and Reh cells were transfected with control shRNA or FIP200 shRNA followed by DNR $(0.4 \mu \mathrm{M})$ treatment in the presence or absence of EP $(5 \mathrm{mM})$ for $24 \mathrm{~h}$. Then, the cells were subjected to western blot analysis of LC3-II/I, p62 and cytosolic HMGB1 expression. Actin was used as a loading control. (D) Jurkat cells were transfected with control or HMGB1 shRNA followed by treatment with DNR $(0.4 \mu \mathrm{M})$ in the presence or absence of EP $(5 \mathrm{mM})$ for $24 \mathrm{~h}$. The cells were subjected to transmission electron microscopy to observe autophagosome-like structures (indicated by the red arrows). (E) Jurkat and Reh cells were treated with DNR $(0.4 \mu \mathrm{M})$ for $24 \mathrm{~h}$ in the presence or absence of EP $(5 \mathrm{mM})$. Then, cell viability was analysed using a CCK-8 kit $\left(\mathrm{n}=3,{ }^{\prime} \mathrm{p}<0.05\right)$. The viability of the control cells was normalised to $100 \%$. Cyt, cytoplasm. Nuc, nucleus. UT, untreated group. 


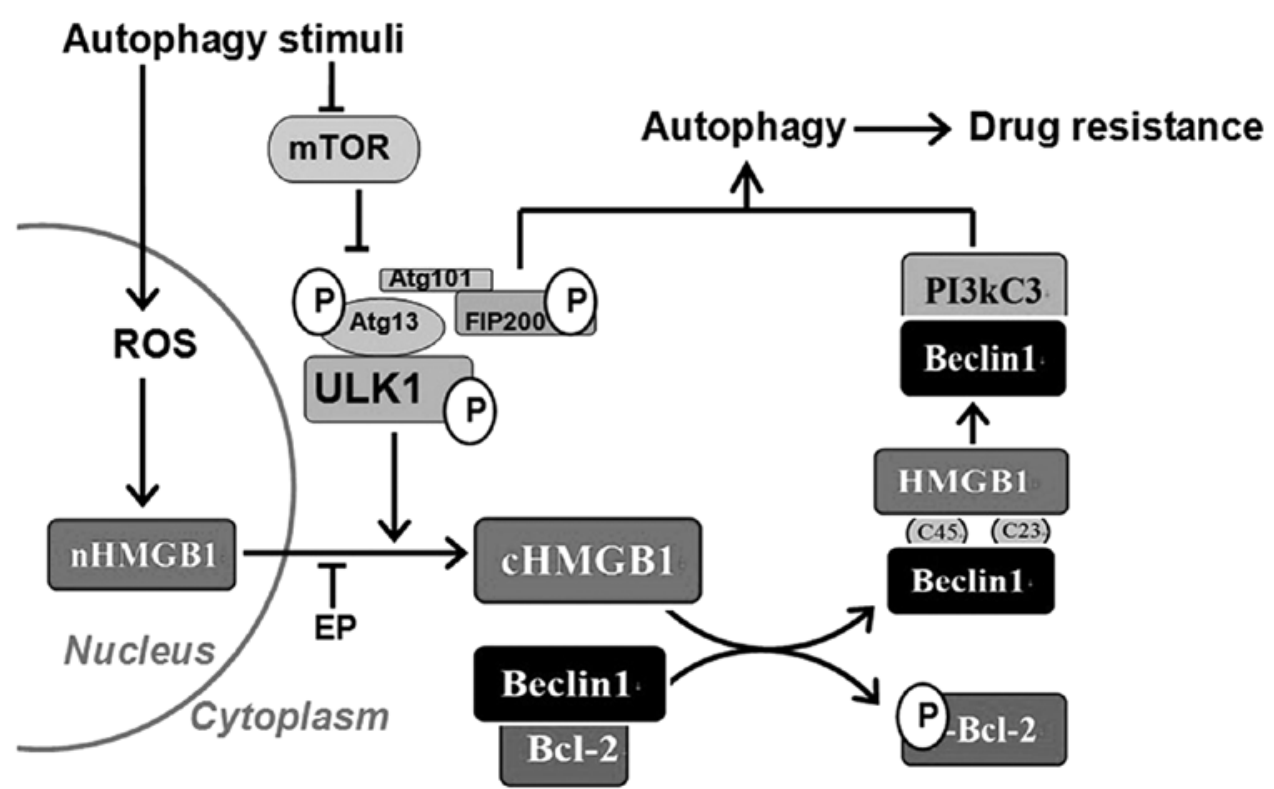

Figure 6. Schematic representation of the potential autophagy-related drug resistance process in leukaemia. Under normal conditions, the ULK1-mAtg13-FIP200 complex is suppressed by mTOR, and Bcl-2 binds to Beclin1 to reduce its pro-autophagic activity. Under stress conditions, HMGB1 translocates to the cytosol, which is promoted by the activation of the upstream complex ULK1-mAtg13-FIP200. Consequently, the Bcl-2-Beclin1 interaction is disrupted by the phosphorylation of Bcl-2 and by competitive displacement of Bcl-2 with HMGB1. The assembly of the HMGB1-Beclin1 complex facilitates the formation of the PI3KC3-Beclin1 complex. These dissociations and recouplings likely enhance the anti-apoptotic state, promote autophagy, and ultimately protect the tumour cells during chemotherapy.

quently complexed with HMGB1 and PI3KC3. In contrast, Beclin1-Bcl-2 was not separated after DNR treatment in the absence of FIP200 or HMGB1. Thus, the HMGB1-Beclin1 and PI3KC3-Beclin1 complexes failed to coalesce. Furthermore, we observed that the depletion of HMGB1 or FIP200 reduced the phosphorylation of $\mathrm{Bcl} 2$ (Fig. 4D), which may result in the stable binding of $\mathrm{Bcl} 2$ to Beclin1.

The translocation of HMGBI is required for autophagymediated chemoresistance in leukaemia cells. Although primarily localised to the nucleus, HMGB1 can translocate to the cytoplasm and the extracellular space during cell activation and cell death. To investigate the role of HMGB1 in autophagy, we focused on its localization. By detecting the expression of HMGB1 in the nucleus and the cytosol separately, we found that the chemotherapy promoted the translocation of HMGB1 from the nucleus to the cytosol in the cultured Jurkat and Reh cells (Fig. 5A). This translocation was also detected using immunofluorescence techniques (Fig. 5B). Remarkably, HMGB1 did not translocate in the absence of FIP200 (Fig. 5A and B). When combining the data of Figs. 4B and $\mathrm{C}$ and $5 \mathrm{~A}$ and $\mathrm{B}$, we concluded that the activation of the ULK1-mAtg13-FIP200 complex induced the translocation of HMGB1 and the subsequent dissociation of Beclin1-Bcl-2 and formation of the HMGB1-Beclin1 complex, ultimately influencing autophagy-related chemoresistance.

As shown in Fig. 5B and C, the Jurkat and Reh cells were treated with DNR in the presence or absence of EP, an inhibitor of HMGB1 translocation. We found that blocking HMGB1 translocation mimicked the knockdown of FIP200 and suppressed autophagy. At the ultrastructural level (Fig. 5D), EP reduced the formation of autophagosomes and autophagolysosomes to a great extent, and this effect was similar to that of HMGB1 or FIP200 depletion (Figs. 3D and 5D). Moreover, in the absence of HMGB1 translocation, autophagy was suppressed, and the leukaemia cells became more sensitive to the chemotherapy (Fig. 5E).

The above results suggested that HMGB1 is involved in the positive regulation and maintenance of autophagy in stressed leukaemia cells and that HMGB1 translocation is a necessary component of the autophagic process. Blocking HMGB1 translocation by interrupting the upstream signal or using a translocation inhibitor may represent a useful strategy for reducing autophagy-induced drug resistance.

\section{Discussion}

Resistance to chemotherapy is an intractable but common barrier to the clinical treatment of leukaemia. The adaptation of leukaemia cells to drug-induced stress in the tumour microenvironment has been proposed to be a key mechanism underlying chemotherapy resistance. Autophagy, a lysosomal degradation process that plays a paradoxical role in anticancer treatment, typically exerts a protective effect by facilitating the acquisition of drug resistance in leukaemia $(9,12,23)$. Consistent with previous reports, our results showed that the detrimental effects of chemotherapy were exacerbated by the inhibition of autophagy (Fig. 2), which indicated that the antitumour agents induced autophagy-related drug resistance. In this study, we further elucidated the crucial function and regulatory relationships of the ULK1-Atg13-FIP200 and HMGB1-Beclin1 complexes. HMGB1 translocation played a crucial role in dissociation of these complexes and in the subsequent complexation.

Vast amount of data have demonstrated that the ULK1Atg13-FIP200 complex integrates incoming autophagic 
signals into autophagosome formation, an essential event for autophagy induction (24-26). FIP200, a key signalling node that regulates various cellular processes, is a central components of the ULK1-Atg13-FIP200 complex and is important for the appropriate phosphorylation of ULK1 $(27,28)$. Wei et al observed that the ablation of FIP200 led to autophagic defects, significantly reduced cell proliferation and inhibited mammary tumourigenesis (29). Liu et al found that the deletion of FIP200 suppressed autophagy and caused osteopenia in mice (30). Bae and Guan reported that the inhibition of autophagy via the inactivation of FIP200 inhibited the repair of damaged DNA and enhanced the efficacy of cancer treatments (31). Consistently, our study showed that silencing FIP200 increased cell death, suppressed autophagy and subsequently sensitised the leukaemia cells to anticancer therapy. FIP200 knockdown achieved a similar effect to that of other pharmacological autophagy inhibitors, such as 3-MA and CQ (Figs. 2 and 3).

HMGB1-Beclin1 is another important complex that is assembled after HMGB1 translocation (13). We examined BMMCs from 38 children and found that HMGB1 was significantly upregulated in the ALL patients who exhibited primary, relapsed or recurrent disease (Fig. 1A) and was equivalent to baseline in the ALL patients who exhibited complete remission. This result was consistent with those of previous studies $(23,32)$ and indicated that HMGB1 contributes to the pathogenesis of leukaemia and even drug resistance. In vitro, we detected HMGB1 translocation from the nucleus to the cytosol in the presence of autophagic stimuli (Fig. 5). Moreover, either the absence of HMGB1 or the inhibition of HMGB1 translocation distinctly attenuated autophagy and ameliorated chemotherapy resistance, and these effects mimicked those of the autophagy inhibitors (Fig. 5). Another autophagic complex component, Beclin1, has been considered as a tumour suppressor (33-35). Reportedly, overexpressing Beclin1 induces significant autophagic cell death in leukaemia (36). However, several investigators measured the Beclin1 expression level in PBMCs or BMMCs from Chinese leukaemia patients and obtained inconsistent results $(37,38)$. More recently, others have disputed the original hypothesis and concluded that Beclin1 mutation might not be significant in human cancer and that Beclin1 is likely not a tumour suppressor gene (39). In our study, no significant difference in Beclin1 expression was detected between the groups (Fig. 1B). This result could be because the decreased Beclin1 expression in leukaemia patient may have been offset by an increase in Beclin 1 expression due to the activation of autophagy. However, the small sample size may reduce the reliability of these results. A long-term study using a greater number of patients is required in the future.

Substantial research has been dedicated to the examination of the functions of the ULK1-mAtg13-FIP200 and HMGB1-Beclin1 complexes, but little attention has been paid to their association. Pan et al recently reported that HMGB1 facilitates the formation of the Beclin-1-PI3K-III complex through activating the MEK/ERK1/2 signalling pathway and then promotes docetaxel resistance in human lung adenocarcinoma (40). Their results support future investigation of HMGB1 as a strategic target. However, they failed to mention the ULK1-mAtg13-FIP200 complex. Huang et al proposed that HMGB1 serves as a downstream signal from ULK1-
mAtg13-FIP200 complex formation and facilitates autophagy by interacting with Beclin1 (41). However, their study only used osteosarcoma cells and did not elucidate the potential mechanism. In the present study, we showed that in leukaemia cells, the ULK1-mAtg13-FIP200 complex functions upstream of HMGB1 and is required for the assembly of the HMGB1Beclin1 and PI3KC3-Beclin1 complexes (Fig. 4). Remarkably, we demonstrated that the ULK1-mAtg13-FIP200 complex partially depended on HMGB1 translocation to facilitate the formation of these downstream complexes (Figs. 4 and 5). This finding is consistent with a recent report on murine pseudomonas infection (42). Moreover, previous reports have suggested that Beclin1 is bound to Bcl-2 under normal conditions but that during autophagy Beclin1 is bound to cytosolic HMGB1 following Bcl-2 phosphorylation (43-46). Our results strongly corroborated this concept (Fig. 4C and D), as these changes were no longer observed after FIP200 or HMGB1 depletion, revealing a potential approach to prevent autophagyrelated drug resistance.

To date, CQ and its derivative hydroxychloroquine (HCQ) are the only autophagy inhibitors that have been used in dozens of clinical trials of human tumours (47). Due to their effectiveness in autophagy inhibition and their anti-angiogenic properties, effects of chemotherapy drugs are clearly enhanced when CQ or HCQ is administered as an adjuvant therapy. However, the clinical application of CQ and HCQ has been limited because of their prolonged half-life, low potency and ocular toxicity. Therefore, the identification of more specific targets and the establishment of safer and more effective therapeutic strategies are highly desirable. Our data provide insight into that which may meet the demand.

In conclusion, by combining previous data with our present results, we have elucidated the process underlying autophagyrelated drug resistance in leukaemia (Fig. 6). The results of the present study suggest that the Ulk1-Atg13-FIP200 complex functions upstream of the HMGB1-Beclin1 and PI3KC3-Beclin1 complexes and HMGB1 translocation is involved in their regulatory relationships. However, this unidirectional regulation model appears to be oversimplified, and additional crosstalk should be considered in future research.

The dissociation and recoupling of autophagic complexes are essential events involved in the autophagy-related chemoresistance. By inhibiting the transformation of these complexes or HMGB1 trafficking, we are likely to render tumour cells more susceptible to conventional therapies and overcome drug resistance in leukaemia.

\section{Acknowledgements}

This study was supported by National Natural Science Foundation of China (grant no. 81100370) and Sun Yat-Sen University Clinical Research 5010 Program (grant no. 2007016).

\section{References}

1. Liu L, Yang M, Kang R, Wang Z, Zhao Y, Yu Y, Xie M, Yin X, Livesey KM, Loze MT, et al: DAMP-mediated autophagy contributes to drug resistance. Autophagy 7: 112-114, 2011.

2. Livesey KM, Tang D, Zeh HJ and Lotze MT: Autophagy inhibition in combination cancer treatment. Curr Opin Investig Drugs 10: 1269-1279, 2009. 
3. White E and DiPaola RS: The double-edged sword of autophagy modulation in cancer. Clin Cancer Res 15: 5308-5316, 2009.

4. Sui X, Chen R, Wang Z, Huang Z, Kong N, Zhang M, Han W, Lou F, Yang J, Zhang Q, et al: Autophagy and chemotherapy resistance: A promising therapeutic target for cancer treatment. Cell Death Dis 4: e838, 2013.

5. Chen S, Rehman SK, Zhang W, Wen A, Yao L and Zhang J: Autophagy is a therapeutic target in anticancer drug resistance. Biochim Biophys Acta 1806: 220-229, 2010.

6. Alers S, Löffler AS, Wesselborg S and Stork B: Role of AMPKmTOR-Ulk1/2 in the regulation of autophagy: Cross talk, shortcuts, and feedbacks. Mol Cell Biol 32: 2-11, 2012.

7. Wong PM, Puente C, Ganley IG and Jiang X: The ULK1 complex: Sensing nutrient signals for autophagy activation. Autophagy 9 124-137, 2013

8. Wirth M, Joachim J and Tooze SA: Autophagosome formation the role of ULK1 and Beclin1-PI3KC3 complexes in setting the stage. Semin Cancer Biol 23: 301-309, 2013.

9. Nencioni A, Cea M, Montecucco F, Longo VD, Patrone F, Carella AM, Holyoake TL and Helgason GV: Autophagy in blood cancers: Biological role and therapeutic implications. Haematologica 98: 1335-1343, 2013.

10. Pyo JO, Nah J and Jung YK: Molecules and their functions in autophagy. Exp Mol Med 44: 73-80, 2012.

11. Viry E, Paggetti J, Baginska J, Mgrditchian T, Berchem G, Moussay E and Janji B: Autophagy: An adaptive metabolic response to stress shaping the antitumor immunity. Biochem Pharmacol 92: 31-42, 2014.

12. Liu L, Yang M, Kang R, Wang Z, Zhao Y, Yu Y, Xie M, Yin X, Livesey KM, Lotze MT, et al: HMGB1-induced autophagy promotes chemotherapy resistance in leukemia cells. Leukemia 25: 23-31, 2011.

13. Tang D, Kang R, Livesey KM, Cheh CW, Farkas A, Loughran P, Hoppe G, Bianchi ME, Tracey KJ, Zeh HJ III, et al: Endogenous HMGB1 regulates autophagy. J Cell Biol 190: 881-892, 2010.

14. Yeo C, Saunders N, Locca D, Flett A, Preston M, Brookman P, Davy B, Mathur A and Agrawal S: Ficoll-Paque versus Lymphoprep: A comparative study of two density gradient media for therapeutic bone marrow mononuclear cell preparations Regen Med 4: 689-696, 2009.

15. Kang R, Tang D, Yu Y, Wang Z, Hu T, Wang $\mathrm{H}$ and Cao L: WAVE1 regulates Bcl-2 localization and phosphorylation in leukemia cells. Leukemia 24: 177-186, 2010.

16. Valizadeh A, Ahmadzadeh A, Teimoori A, Khodadadi A and Saki G: Effects of TNF secreting HEK cells on B lymphocytes' apoptosis in human chronic lymphocytic leukemias. Asian Pac J Cancer Prev 15: 9885-9889, 2014.

17. Mizushima N and Yoshimori T: How to interpret LC3 immunoblotting. Autophagy 3: 542-545, 2007

18. Tang D, Kang R, Xiao W, Jiang L, Liu M, Shi Y, Wang K, Wang H and Xiao X: Nuclear heat shock protein 72 as a negative regulator of oxidative stress (hydrogen peroxide)-induced HMGB1 cytoplasmic translocation and release. J Immunol 178: 7376-7384, 2007.

19. Klionsky DJ, Abdalla FC, Abeliovich H, Abraham RT, AcevedoArozena A, Adeli K, Agholme L, Agnello M, Agostinis P, Aguirre-Ghiso JA, et al: Guidelines for the use and interpretation of assays for monitoring autophagy. Autophagy 8: 445-544, 2012.

20. Ylä-Anttila P, Vihinen H, Jokitalo E and Eskelinen EL: Monitoring autophagy by electron microscopy in mammalian cells. Methods Enzymol 452: 143-164, 2009.

21. Zhang XJ, Chen S, Huang KX and Le WD: Why should autophagic flux be assessed? Acta Pharmacol Sin 34: 595-599, 2013

22. Mizushima N, Yoshimori T and Levine B: Methods in mammalian autophagy research. Cell 140: 313-326, 2010.

23. Yang L, Yu Y, Kang R, Yang M, Xie M, Wang Z, Tang D, Zhao M, Liu L, Zhang H, et al: Up-regulated autophagy by endogenous high mobility group box-1 promotes chemoresistance in leukemia cells. Leuk Lymphoma 53: 315-322, 2012.

24. Alers S, Löffler AS, Paasch F, Dieterle AM, Keppeler H, Lauber K, Campbell DG, Fehrenbacher B, Schaller M, Wesselborg S, et al: Atg13 and FIP200 act independently of Ulk1 and Ulk2 in autophagy induction. Autophagy 7: 1423-1433, 2011.

25. Jung CH, Jun CB, Ro SH, Kim YM, Otto NM, Cao J, Kundu M and Kim DH: ULK-Atg13-FIP200 complexes mediate mTOR signaling to the autophagy machinery. Mol Biol Cell 20 1992-2003, 2009.

26. Ganley IG, Lam H, Wang J, Ding X, Chen S and Jiang X: ULK1. ATG13.FIP200 complex mediates mTOR signaling and is essential for autophagy. J Biol Chem 284: 12297-12305, 2009.
27. Gan B and Guan JL: FIP200, a key signaling node to coordinately regulate various cellular processes. Cell Signal 20: 787-794, 2008.

28. Hara T, Takamura A, Kishi C, Iemura S, Natsume T, Guan JL and Mizushima N: FIP200, a ULK-interacting protein, is required for autophagosome formation in mammalian cells. J Cell Biol 181: 497-510, 2008.

29. Wei H, Wei S, Gan B, Peng X, Zou W and Guan JL: Suppression of autophagy by FIP200 deletion inhibits mammary tumorigenesis. Genes Dev 25: 1510-1527, 2011

30. Liu F, Fang F, Yuan H, Yang D, Chen Y, Williams L, Goldstein SA, Krebsbach PH and Guan JL: Suppression of autophagy by FIP200 deletion leads to osteopenia in mice through the inhibition of osteoblast terminal differentiation. J Bone Miner Res 28 2414-2430, 2013.

31. Bae H and Guan JL: Suppression of autophagy by FIP200 deletion impairs DNA damage repair and increases cell death upon treatments with anticancer agents. Mol Cancer Res 9: $1232-1241,2011$

32. Kang R, Tang DL, Cao LZ, Yu Y, Zhang GY and Xiao XZ: High mobility group box 1 is increased in children with acute lymphocytic leukemia and stimulates the release of tumor necrosis factor-alpha in leukemic cell. Zhonghua Er Ke Za Zhi 45: 329-333, 2007 (In Chinese)

33. Liang XH, Jackson S, Seaman M, Brown K, Kempkes B, Hibshoosh $\mathrm{H}$ and Levine B: Induction of autophagy and inhibition of tumorigenesis by beclin 1. Nature 402: 672-676, 1999.

34. Aita VM, Liang XH, Murty VV, Pincus DL, Yu W, Cayanis E, Kalachikov S, Gilliam TC and Levine B: Cloning and genomic organization of beclin 1, a candidate tumor suppressor gene on chromosome 17q21. Genomics 59: 59-65, 1999.

35. Yue Z, Jin S, Yang C, Levine AJ and Heintz N: Beclin 1, an autophagy gene essential for early embryonic development, is a haploinsufficient tumor suppressor. Proc Natl Acad Sci USA 100: 15077-15082, 2003.

36. Tong Y, You L, Liu H, Li L, Meng H, Qian Q and Qian W: Potent antitumor activity of oncolytic adenovirus expressing Beclin-1 via induction of autophagic cell death in leukemia. Oncotarget 4: 860-874, 2013

37. Hu XY, Bai H, Pan YZ, Wnag CB, Wu B, Zhao Q, Ai H, Chen Z and Han X: Expression of autophagy related gene Beclin1 and MAPLC3 in bone marrow mononuclear cells isolated from acute leukemia patients and its significance. Zhongguo Shi Yan Xue Ye Xue Za Zhi 19: 598-601, 2011 (In Chinese).

38. Wan SY, Zhang R, Wang YY, Cen JN, Zhou J, Yang Y, Jiang F and Chen ZX: Expression of autophagy related gene Beclin1 in myelodysplastic syndrome patients and its significance. Zhongguo Shi Yan Xue Ye Xue Za Zhi 21: 936-939, 2013 (In Chinese).

39. Laddha SV, Ganesan S, Chan CS and White E: Mutational landscape of the essential autophagy gene BECN1 in human cancers. Mol Cancer Res 12: 485-490, 2014.

40. Pan B, Chen D, Huang J, Wang R, Feng B, Song H and Chen L: HMGB1-mediated autophagy promotes docetaxel resistance in human lung adenocarcinoma. Mol Cancer 13: 165, 2014

41. Huang J, Ni J, Liu K, Yu Y, Xie M, Kang R, Vernon P, Cao L and Tang D: HMGB1 promotes drug resistance in osteosarcoma. Cancer Res 72: 230-238, 2012.

42. Li Y, Gan CP, Zhang S, Zhou XK, Li XF, Wei YQ, Yang JL and Wu M: FIP200 is involved in murine pseudomonas infection by regulating HMGB1 intracellular translocation. Cell Physiol Biochem 33: 1733-1744, 2014.

43. Marquez RT and Xu L: Bcl-2:Beclin 1 complex: multiple, mechanisms regulating autophagy/apoptosis toggle switch. Am J Cancer Res 2: 214-221, 2012.

44. Kang R, Zeh HJ, Lotze MT and Tang D: The Beclin 1 network regulates autophagy and apoptosis. Cell Death Differ 18: 571-580, 2011.

45. Saeki K, Yuo A, Okuma E, Yazaki Y, Susin SA, Kroemer G and Takaku F: Bcl-2 down-regulation causes autophagy in a caspaseindependent manner in human leukemic HL60 cells. Cell Death Differ 7: 1263-1269, 2000.

46. Wei Y, Pattingre S, Sinha S, Bassik M and Levine B: JNK1mediated phosphorylation of Bcl-2 regulates starvation-induced autophagy. Mol Cell 30: 678-688, 2008.

47. Amaravadi RK, Lippincott-Schwartz J, Yin XM, Weiss WA, Takebe N, Timmer W, DiPaola RS, Lotze MT and White E: Principles and current strategies for targeting autophagy for cancer treatment. Clin Cancer Res 17: 654-666, 2011. 